\title{
El sentido del trabajo en la sociedad digital: lecciones del crowdsourcing'
}

\section{The Meaning of Work in the Digital Society: Lessons from the Crowdsourcing}

\author{
ALESSANDRO GENTILE \\ Profesor de Sociología \\ Facultad de Ciencias Sociales y del Trabajo \\ Universidad de Zaragoza \\ agentile@unizar.es
}

\section{Resumen}

En el artículo exploramos la experiencia del crowdsourcing, como actividad laboral y colaborativa novedosa dentro de redes altamente especializadas de productores, distribuidores y consumidores. Esta forma de entender, organizar y plasmar el trabajo a través de plataformas colaborativas está todavía poco estudiada: en particular, queda pendiente conocer bien cómo se articulan entre sí la dimensión instrumental-económica de este tipo de trabajo y su dimensión expresivaidentitaria, teniendo en cuenta la participación voluntaria de los actores involucrados (empresas y profesionales, además de potenciales clientes). Abordamos ambos aspectos desde una perspectiva relacional para entender lo que el crowdsourcing ofrece en términos de construcción de valor, de gestión del conocimiento experto y de fomento de nuevos sujetos colectivos.

Palabras claves: TICs, Plataformas Digitales, Economía Colaborativa, Trabajo Relacional

\section{PABLO GARCÍA RUÍZ}

Departamento de Sociología

Universidad de Zaragoza

pgruiz@unizar.es

\section{Abstract}

In the article we explore the use of crowdsourcing as an innovative working and collaborative tool within the highly-specialized networks of producers, distributors and consumers. This way of understanding, organizing and broadcasting work by means of collaborative platforms has not been studied in depth. Specifically what is yet to be known is how the instrumental-economic along with the expressiveidentitarian dimensions of this type of work are constructed, keeping in mind the voluntary participation of all actors involved (businesses and professions, as well as potential clients). We broach both aspects from a relational perspective in order to understand what crowdsourcing offers in terms of constructing value, managing expert knowledge and promoting new collective subjects.

Keywords: ICTs, Digital Platforms, Collaborative Economy, Relational Labour

1 Este artículo se ha desarrollado en el marco del Proyecto de Investigación "La Restructuración de la Gobernanza del Estado del Bienestar: determinantes políticos e implicaciones sobre la (des) mercantilización de los riesgos" del Plan Nacional I+D+i 2018-2020 (CSO2017-85598-R). 


\section{INTRODUCCIÓN}

La cultura de la sociedad posmoderna de los países del capitalismo avanzado se enriquece cada vez más con nuevas formas de entender, organizar y planificar el trabajo. Este proceso conlleva unos cambios inéditos para las organizaciones productivas, para los profesionales y para los sistemas de empleo cuyas consecuencias aún no advertimos plenamente. Por una parte, las nuevas tecnologías ligadas a la inteligencia artificial están ampliando los tipos, los contenidos y los estilos de trabajo ${ }^{2}$. La digitalización y la robotización la economía dan lugar a la sustitución de personas por máquinas en los procesos productivos a nivel global ${ }^{3}$ a la aparición de trabajos virtuales propios de una economía posmoderna caracterizada por la revolución 4.0 en todas las empresas, especialmente en las más competitivas e innovadoras.

Por otra parte, con cada vez menos frecuencia el trabajo se define como una realidad de naturaleza instrumental y abstracta, ni se mide principalmente en tiempo cuantitativo. Por el contrario, genera crecientes expectativas de autonomía, flexibilidad y creatividad, y se valora según una percepción más cualitativa del tiempo empleado y de todos los contenidos que aporta. A la par de ello, las nuevas formas de organizar el trabajo implican una mayor incertidumbre y precariedad para los actores que lo llevan a cabo, con crecientes riesgos de inestabilidad e inseguridad.

El cambio cultural y el cambio estructural de la actividad laboral son procesos interrelacionados que generan una nueva subjetividad de la acción laboral además de significativas novedades en las formas organizativas ${ }^{4}$.

Un ejemplo sobresaliente del nuevo tipo de interrelaciones creativas que aglutinan en sí estos mismos cambios es el crowdsourcing, una convocatoria abierta para que un grupo de personas complete unas determinadas tareas de forma voluntaria, utilizando sus propios recursos ${ }^{5}$. Las organizaciones emplean el crowdsourcing para actividades tan diferentes como la generación de ideas, la resolución de problemas, la discusión, evaluación y selección de soluciones y la producción de bienes y servicios con alto valor añadido. El funcionamiento de este nuevo modelo de trabajo es relativamente sencillo: un nú-

2 Brynjolfsson, E. y McAfee, A. (2014).

McKinsey (2017).

Donati, P. (2017); Hidalgo, M. A. (2018).

5 Howe, J. (2006); Estellés-Arolas, E. y González-Ladrón-de-Guevara, F. (2012); Goldman, B. L. (2014); Kietzmann, J. H. (2017). 
mero variable de personas anónimas responden a la llamada y aportan su contribución concreta, ideas, tiempo e incluso dinero sin tener siempre asegurada una compensación a cambio. Algunas empresas organizan concursos y ofrecen premios motivadores, pero otras no suelen hacerlo. Ninguna de estas convocatorias tiene garantizado el éxito: algunas empresas consiguen reunir un número suficiente y eficaz de participantes para la actividad que persiguen; otras en cambio no logran movilizar a la gente, dejando que las tareas se llevan a cabo o, aún peor, en el caso de que logren movilizar unos participantes, acaban generando resultados contrarios a los que se esperaban.

Observando estas dinámicas nos planteamos algunas cuestiones importantes: ¿Qué motivos llevan a la gente a implicarse en proyectos de crowdsourcing? ¿Qué expectativas se crean entre los participantes y la organización? Entender cómo una llamada abierta puede aprovechar el trabajo de una multitud amplia y posiblemente heterogénea de personas y profesionales requiere comprender no solo sus motivaciones, más allá de la compensación económica, sino también el tipo de relaciones que se gestan entre ellos, y que les conecta/vincula/compromete con la organización convocante.

En este artículo estudiamos el crowdsourcing como una actividad emergente y muy importante dentro de unas redes altamente diferenciadas de productores, distribuidores y consumidores ${ }^{6}$. Nos proponemos explorar las actividades de crowdsourcing como un campo en el que las dimensiones instrumentales y transaccionales del trabajo compiten y se entrelazan con aquellos aspectos más expresivos e identitarios que atañen a los participantes. Por ello, en términos metodológicos, en el artículo describimos algunos de los más destacados casos empresariales de crowdsourcing a nivel internacional; acto seguido, proponemos la teoría relacional ${ }^{7}$ como marco de investigación para entender mejor este fenómeno, eso es, para conocer las relaciones sociales sobre las cuales el crowdsourcingse fundamenta y las motivaciones que activan estas mismas relaciones, según unos principios de participación, mutualidad y autorrealización.

\footnotetext{
6 Schwartz, D. (2018).

7 Donati, P. (2006; 2010);Donati, P. y Archer, M. (2015); Donati, P. y García Ruiz, P. (2021).
} 


\section{ESTADO DE LA CUESTIÓN: LA EMERGENCIA DEL CROWDSOURCING}

El crowdsourcing se puede describir como un tipo de actividad participativa en la que un individuo, una institución, una organización sin fines de lucro o una empresa comercial proponen a un grupo de personas de diferentesramas de conocimiento y especialización, heterogeneidad y número, a través de una llamada abierta y flexible, la realización voluntaria de unas tareas productivas. La complejidad de las funciones a desarrollar es variable y modular porque cada uno que esté interesado y se ofrezca para realizarlas, tendrá que participar aportando concretamente su propio trabajo, dinero, conocimiento y experiencia ${ }^{8}$. Para aclarar estos mecanismos podemos hacer referencia a Threadless, una compañía de camisetas de Internet, cuyos diseños son creados y seleccionados por los usuarios; ModCloth, una tienda de ropa en línea que permite a sus usuarios opinar y votar por diseños de ropa antes de que se pongan a la venta; y FiatMio, una iniciativa lanzada por esta grande empresa de automóviles en Brasil a través de la cual se creó un modelo de coche siguiendo las sugerencias de usuarios y potenciales clientes.

En 2008 la cadena de cafeterías Starbucks pone en marcha la plataforma on-line "My Starbucks Idea" 9 . Su propósito es recoger, compartir y considerar estratégicamente los gustos y las opiniones de sus clientes para mejorar sus productos, el ambiente y la decoración de sus tiendas, proponiendo nuevos conceptos empresariales que fueran adecuados a la experiencia de los destinatarios últimos del servicio ofrecido. El principio básico de esta iniciativa era que los clientes de Starbucks (más de 50 millones en todo el mundo) son los que conocen con más precisión la calidad de sus productos porque los consumen habitualmente. Por tanto, ellos saben mejor que nadie qué les gustaría añadir a la experiencia Starbucks, cómo mejorarla o qué cambios incorporar a los productos. Así, los clientes se convierten en una fuente fundamental de información e innovación para la compañía. Los usuarios que quieren participar en este proyecto deben registrarse en MyStarbucksIdea.com y enviar una sugerencia. Ellos también podían comentar y votar las sugerencias de otros usuarios. Así surgió un ranking de las propuestas más populares que, acto seguido, venían evaluadas por un equipo de expertos, empleados de Starbucks, para identificarlas mejores sugerencias y proponer su implementación. Desde

8 Brabham, D. C. (2010); Estellés-Arolas, E. y González-Ladrón-de-Guevara, F. (2012).

$9 \operatorname{ICEMD}(2018)$. 
entonces la plataforma recibió más de 190.000 ideas, de las cuales más de 300 fueron insertadas en el plan estratégico de la compañía.

Acudir a las masas para obtener ideas, opiniones y aportaciones no es algo nuevo. En 1714, el gobierno británico ofreció un premio monetario -conocido como el Premio de Longitud- por la mejor forma de medir la posición longitudinal de un barco. En 1916, Planters Peanuts organizó un concurso público de diseño de logotipos a través del cual se creó el Sr. Peanut. En 1936, el logotipo de Toyota fue diseñado por medio de un concurso similar, como lo fue la arquitectura de la Ópera de Sydney en $1955^{10}$. Lo que quizá resulta novedoso es que las organizaciones están utilizando el conocimiento (que es amplio y al tiempo disperso en la sociedad) como un recurso muy útil para sus operaciones ordinarias de producción, marketing y venta. Crowdsourcing parece ser el resultado de "tomar una función que solían realizar los empleados y subcontratarla a una red indefinida (y generalmente grande) de personas en forma de una convocatoria abierta" ${ }^{11}$. Se trata de un mecanismo estratégico que permite a las organizaciones "beneficiarse de un gran número de personas que aportan diversidad en ideas, conocimiento y experiencia, que se aprovecha en beneficio de la organización"12.

El fabricante farmacéutico Eli Lilly financió el lanzamiento de InnoCentive en 2001 como una forma de conectarse con el talento y los conocimientos de personas ajenas a la empresa, que podrían ayudar a desarrollar medicamentos y acelerar su comercialización. Desde el principio, InnoCentive abrió las puertas a otras empresas deseosas de acceder al "tesoro" de expertos ad hoc de la red. Empresas como Boeing, DuPont y Procter \& Gamble ahora publican sus problemas científicos más importantes en el sitio web de InnoCentive; cualquier persona en la red de InnoCentive puede tomar una oportunidad para descifrarlos ${ }^{13}$. Los resultados son bastante buenos: hasta ahora, más del 30\% de los problemas publicados en el sitio han sido solucionados, una cifra mucho mayor que la obtenida mientras se utilizaba un enfoque interno tradicional.

Las actividades de crowdsourcing transcurren online, pero no de manera exclusiva. Por ejemplo, cuando Peter Jackson, director de la película El señor

10 Kietzmann, J. H. (2017), p. 151.

11 Howe, J. (2006), p.1.

12 Wilson, M.; Robson, K. y Botha, E. (2017), p. 247.

13 Howe, J. (2006), p. 3 
de los anillos: Las dos torres, necesitó crear el sonido de los ejércitos de orcos en combate, recurrió a una multitud física en lugar de virtual: En el estadio Westpac en Wellington, Nueva Zelanda, Jackson pidió a 20.000 fanáticos de los grillos gritar, gritar y gruñir para crear el sonido de los grupos de Uruk-hai reunidos antes de la Batalla de Helms Deep ${ }^{14}$. El crowdsourcing puede ayudar a las empresas a aprovechar recursos que de otra manera serían inalcanzables para obtener una amplia gama de objetivos, como crear una ventaja competitiva, superar el pensamiento grupal y aprovechar al máximo el conocimiento disperso ${ }^{15}$.

\section{ANÁLISIS DESCRIPTIVO: UNA EXTENSA VARIEDAD DE ACTIVIDADES}

Las organizaciones emplean crowdsourcing para diferentes tipos de actividades tales como generar ideas, resolver problemas, evaluar y seleccionar propuestas innovadoras, dinamizar foros de discusión, votar preferencia por las diferentes alternativas propuestas, pronosticar el éxito del producto o también de la producción y de la financiación ${ }^{16}$. Una de las acciones más frecuentes es la comercialización participativa (co-creación) de productos o mensajes comerciales ${ }^{17}$. A través de este proceso, una organización invita opiniones para preguntas con complejidad variable, según el caso, y luego evalúa las ideas propuestas. La co-creación describe un proceso en el cual los individuos llevan a cabo sus iniciativas contando con las contribuciones de los demás a través del intercambio, la combinación creativa de sus conocimientos ${ }^{18}$. Por ejemplo, la compañía de cerveza Heineken ha puesto en práctica varias iniciativas para aprovechar la libre contribución de sus consumidores ${ }^{19}$. Una de ellas, titulada "Your Heineken", invitaba a los clientes a participar en el diseño de las botellas de cerveza. Otra, titulada "Ideas Brewery", buscaba ideas que sirvan para "hacer más verdes" sus productos, reciclando sus envases actuales, para encontrar nuevos materiales de envases sostenibles o incluso formas de transporte más eficientes y menos contaminantes. Tras esta "lluvia de ideas", la compañía elige las 100 propuestas que mejoran con aportaciones de otros

\footnotetext{
14 Prpi , J.; Shukla, P. P.; Kietzmann, J. H. y McCarthy, I. P. (2015), p. 78.

15 Prpi , J.; Shukla, P. P.; Kietzmann, J. H. y McCarthy, I. P. (2015), p. 77.

16 Fedorenko, I.; Berthon, P. y Rabinovich, T. (2017).

17 Brunswicker,S.; Bilgram, V. y Fueller, J. (2017).

18 De Masi, D. (2002).

$19 \operatorname{ICEMD}(2018)$.
} 
usuarios y de los profesionales de Heineken. Finalmente, la empresa designa aquellas ideas que se han de poner en práctica para mejorar la oferta de su producto.

Otra forma frecuente de crowdsourcing es la financiación de proyectos, sea de entidades sociales como también de compañías de negocios. Esta modalidad es conocida como crowdfounding. Ha sido muy utilizada para actividades de microcrédito, voluntariado y emprendimiento social, pero también existe en el ámbito del mercado. Algunas compañías se dirigen a los potenciales consumidores de un nuevo producto y les piden que colaboren, aportando la financiación necesaria para su desarrollo y lanzamiento. Es este el caso de empresas que, generalmente, no cuentan con recursos suficientes y confían en vender una idea suficientemente atractiva como para que haya quienes están dispuestos a pagar por algo que aún no existe.

Otras organizaciones han empezado a usar sitios web de crowdfounding no solo como fuente de financiación, sino también como plataformas de marketing para garantizar una cartera completa de productos, impulsar la imagen de marca y obtener apoyo para las causas sociales que pueden estar relacionadas con la marca ${ }^{20}$. Un buen ejemplo de ello es el Opal, la máquina de cubitos de hielo tipo "nugget (pepitas)", desarrollada por General Electric Appliances (GE) con la colaboración de las plataformas en línea FirstBuild e Indigogo $^{21}$. La primera es una comunidad de co-creación que está cambiando la forma en que los productos llegan al mercado: en Opal la contribución de FirstBuild consistió en una presencia virtual online y en una "microfactoría" física equipada con las herramientas necesarias para fabricar prototipos de productos. GE decidió usar FirstBuild como banco de pruebas, preguntándose si sería posible satisfacer más fácilmente el antojo que muchas personas tienen por un producto nuevo, como un tipo particular de hielo. La mayoría de los cubitos de hielo son bloques de agua congelados de varios tamaños y formas. "Nugget-ice" es algo diferente. Sus pequeños trozos en forma de barril son porosos y se semi-congelan. Estas cualidades permiten que el hielo absorba bien los sabores y que sea más fácil de masticar, que aparentemente es lo que algunas personas quieren, y mucho. Debido a que hacer hielo de pepita es más complejo que congelar agua, las máquinas que lo producen cuestan varios miles de dólares, un precio inaccesible para la mayoría de los hogares. GE que-

20 Kietzmann, J. H. (2017), p. 153.

21 Brynjolfsson, E. y McAfee, A. (2017), pp. 16-19. 
ría ver si los usuarios de FirstBuild podrían diseñar y crear prototipos de una fábrica de hielo tipo pepita, adaptado a las capacidades de gasto de los hogares, y se lanzó una convocatoria online en 2015. El ganador fue Ismael Ramos, un diseñador de Guadalajara (México), cuya entrada de diseño 'Stone Cold' visualizó una máquina cúbica adecuada para encimeras de cocina, con un cubo de hielo de plástico transparente extraíble. Ramos recibió como premio $\$ 2.000$ y una de las primeras versiones de su creación. Hubo dos finalistas en el concurso, que también recibieron premios en efectivo. Las personas de la micro factoría FirstBuild comenzaron a fabricar y refinar prototipos dela máquina de cubitos de hielo. Durante todo el tiempo, estuvieron en contacto con la comunidad en línea que se había formado alrededor del proyecto, haciendo preguntas sobre cómo debería verse el cubo de hielo extraíble, cómo detectar cuándo estaba lleno, si la máquina debería incluir una pala para recoger los cubitos, etc.

La máquina estaba preparada, pero aún quedaba por ver si tendría un mercado suficiente. Para despejar ese interrogante, GE recurrió a Indiegogo, una comunidad online de crowdfounding cuyos miembros no son simples inversores o prestamistas, aunque con frecuencia esperan algún tipo de recompensa. A través de esta otra plataforma, GE quería saber si habría mercado para algo tan raro como una máquina de hielo de mascar. La crowd respondió positivamente, hizo aportaciones económicas como pre-pago de Opal, una máquina que aún no estaba fabricada. GE no necesitaba el dinero, pero sí el conocimiento sobre un posible mercado futuro que hasta entonces era altamente incierto. En su colaboración con FirstBuild e Indiegogo, GE había desplegado una nueva estrategia para aprovechar muchas mentes que no estaban en su nómina, así como un mercado para su máquina de hielo.

Ejemplos como los de Opal, Heineken, Starbucks and InnoCentive muestran que, mediante el crowdsourcing, las empresas se benefician de la colaboración (ideas, tiempo, esfuerzo) de personas que no están vinculadas a ellas. La frontera entre empleados y no empleados se desdibuja. Además, en este proceso surgen nuevas empresas, como Indiegogo and FirstBuild, cuyo objeto es facilitar a otras empresas el acceso al talento y los recursos de la crowd ${ }^{22}$.

22 Kietzmann, J. H. (2017); Täuscher, K. (2017). 
$\mathrm{Al}$ utilizar un "proveedor de servicios de crowdsourcing", la empresa no tiene que ocuparse de todos los problemas estratégicos, logísticos y legales relacionados con una iniciativa de este tipo ${ }^{23}$. El crowdsourcing se convierte en una actividad que beneficia a la empresa que necesita colaboración, a la plataforma que facilita la participación, y a los propios miembros de la comunidad que se genera en torno a la plataforma. Los modelos comerciales de crowdsourcing ofrecen comunidades en línea administradas para los miembros de la multitud. Skillshare, por ejemplo, ofrece una plataforma digital que ofrece miles de clases en vivo sobre una amplia variedad de temas. En lugar de empleados, utiliza expertos independientes para impartir clases relacionadas con sus conocimientos e intereses. Los profesores en línea de Skillshare pueden intercambiar ideas y mejores prácticas sobre temas tales como la estructura de conferencias o cómo tratar con estudiantes difíciles gracias a estos canales comunitarios. La variedad y la relevancia del público crean el contenido, se atraen a clientes como Twitter o Adobe que adoptan el servicio para capacitar a sus empleados ${ }^{24}$.

\section{EL ÉXITO NO ES SEGURO}

El Crowdsourcing parece ofrecer unas oportunidades y unos beneficios claros a todos los participantes. Sin embargo, no todas las convocatorias salen bien. A veces, la causa del fracaso reside en que la crowd a la que se apela, de hecho, no existe. "Las multitudes deben construirse, casi nunca preexisten"25. Diferentes multitudes poseen diferentes conocimientos, habilidades u otros recursos y, en consecuencia, pueden aportar diferentes tipos de valor a una organización. Más específicamente, se trata de buscar comunidades de interés con individuos caracterizados por sensibilidades, competencias y/o intereses similares ${ }^{26}$, pero éstos no siempre se conocen entre sí o han colaborado juntos en un mismo proyecto. Puede ocurrir, entonces, que no haya gente suficiente con los conocimientos o recursos buscados.

También puede ocurrir que la empresa no sepa movilizar adecuadamente a la crowd. A veces, en lugar de colaborar con las operaciones y metas de la em-

23 de Beer, J.; McCarthy, I. P.; Soliman, A. y Treen, E. (2017), p. 215.

24 Para obtener acceso a toda la gama de clases, los alumnos pagan una tarifa mensual. Como recompensa por su contribución, Skillshare paga una regalía a sus miembros de la multitud, dependiendo de la cantidad de estudiantes que puedan atraer a sus clases en vivo. Tauscher, K. (2017), p. 238.

25 Prpi , J.; Shukla, P. P.; Kietzmann, J. H. y McCarthy, I. P. (2015), p. 80.

26 Brabham, D. C. (2010). 
presa, la crowd reacciona de manera inesperada o incluso dañina para la empresa que la convocó. Las mentes colectivas y la colaboración no necesariamente generan buenas ideas y es posible que las multitudes reaccionen a las iniciativas burlándose de ellas y promoviendo ideas alternativas o contrarias. La intención de Chevrolet de actualizar su modelo Tahoe fue un buen ejemplo de una llamada con resultados no deseados ${ }^{27}$. El Chevrolet Tahoe fue uno de los vehículos más populares en Estados Unidos en 2005, con más de 150.000 unidades vendidas. Después de años sin mejoras importantes, el Tahoe se modificó y en marzo de 2006 el equipo de marketing de Chevrolet ideó un concurso en línea para promover su nuevo lanzamiento. Los concursantes fueron invitados a desarrollar anuncios a través de videos de 30 segundos para mostrarles nuevas características del Tahoe. Para alentar las presentaciones, la empresa creó un microsite dedicado a la campaña (Chevyapprentice.com) que albergaba una herramienta sencilla de edición multimedia, así como música y videos que eran proporcionados por la misma empresa. Los usuarios tenían la capacidad de editar el material y agregar sus propios subtítulos, luego se les animó a compartir los videos en YouTube y otras plataformas de redes sociales. La respuesta de los usuarios fue impresionante: hubo más de 30.000 entradas durante las cuatro semanas de concurso. La mayoría de los videos creados por los participantes comunicaron los aspectos positivos del Tahoe, como sus asientos retráctiles y su nuevo diseño. Pero casi de inmediato aparecieron unos videos que denunciaban el impacto del vehículo en el medioambiente. Los participantes conscientes de este problema aprovecharon la oportunidad de criticar a Tahoe por su consumo excesivo de combustible y su contribución al calentamiento global. Algunas de las presentaciones de los consumidores incluyeron referencias también a otros temas controvertidos como la guerra de Irak y las afiliaciones religiosas de los conductores.

Aprovechar la creatividad de una multitud puede proporcionar un medio altamente eficiente y efectivo de adquirir ideas, trabajos y contenidos para resolver problemas. Pero las soluciones de crowdsourcing también pueden implicar unos riesgos, que se adscriben a malas conductas, publicidades negativas y consecuencias legales asociadas con la propiedad intelectual ${ }^{28}$.

A veces, la multitud reacciona de manera inesperada y se rebela contra la empresa, no por sus valores o por su imagen sino porque percibe el trato como injusto. Moleskine, una popular marca de artículos de papelería, se asoció con

27 Wilson, M.; Robson, K. y Botha, E. (2017), pp. 249-250.

28 Fedorenko, I.; Berthon, P. y Rabinovich, T. (2017). 
DesignBoom, una agencia de publicidad, para crear una amplia serie de diseños de un logotipo para su $\operatorname{blog}^{29}$. Moleskine ofreció un premio de $7.000 €$ por el mejor diseño, pero retuvo los derechos de propiedad de todos los envíos. Solo un ganador se beneficiaría, pero todos los participantes perderían la propiedad de sus diseños sin compensación. La comunidad reclutada se quejó en Moleskine por lo que se percibió como una especulación sobre los derechos de propiedad intelectual de los fanáticos creativos de la marca. Moleskine se defendió de las críticas recibidas en Facebook alegando que no era la única organización que participaba en dicha estrategia y que los contribuyentes no estaban obligados a participar si consideraban que las condiciones eran injustas. Esta respuesta alimentó más protestas que, finalmente, obligaron a la empresa a disculparse públicamente.

Las empresas de crowdsourcing requieren más que una plataforma en línea y algún tipo de oferta de premios. En concreto, para ser exitosos en este tipo de iniciativas, se necesita una comprensión de las motivaciones de los participantes, si quieren evitar que sus intentos de aprovechar la sabiduría de la multitud conduzcan a consecuencias desagradables y resulten contraproducentes.

\section{MOTIVOS PARA PARTICIPAR}

La transformación del trabajo en la sociedad posmoderna está dando lugar a la aparición de nuevas formas de trabajo, que responden a las crecientes expectativas de innovación, autonomía y flexibilidad ${ }^{30}$, pero también implican una mayor incertidumbre y más riesgos ${ }^{31}$. La literatura sobre historia social del trabajo muestra que en la actualidad estamos asistiendo a un proceso de diferenciación de la cultura del trabajo ${ }^{32}$, están surgiendo modos diversos de dar significados al trabajo y de valorarlo como eje central en la existencia de los sujetos implicados.

En la sociedad capitalista moderna, el trabajo se entiende fundamentalmente como "tiempo a cambio de salario": las personas realizan tareas para la producción y distribución de bienes y servicios que las empresas remuneran

29 de Beer, J.; McCarthy, I. P.; Soliman, A. y Treen, E. (2017), p. 208.

30 Castells, M. (2005).

31 Donati, P. (2017); Hines, A. (2011); Hodgson, G. M. (2016).

32 Kallash, S. yKruse, M.(2012); Lips-Wiesme, M.y Morris, L. (2017); Rosso, B. D.; Dekas, K. H. y Wrzesniewski, A. (2010). 
con los beneficios que obtienen de su venta. La teoría relacional ${ }^{33}$ sugiere que es más fácil comprender el sentido del trabajo si se abandona el paradigma funcional, que define el trabajo como transacción, y se adopta una perspectiva que lo define como relación social normativa y culturalmente establecida.

El crowdsourcing ofrece un interesante escenario para esta observación relacional de un trabajo que, de hecho, ya no se ajusta al paradigma transaccional de la sociología moderna del trabajo ${ }^{34}$. La idea de transacción indica un intercambio específico: se da algo valioso a cambio de algo también valioso. Sin embargo, la crowd con frecuencia no se comporta así. Mucha gente aporta sus recursos, sus ideas y su tiempo, en el marco de una ganancia que debe ser mutua entre todos los participantes: los miembros del crowd recibirán siempre algo (explícito o implícito) a cambio de su disponibilidad, que no necesariamente corresponderá a una remuneración de tipo económica ${ }^{35}$. Entonces, ¿Qué motivos llevan a la gente a responder a una call y usar sus recursos para realizar una tarea que beneficiará a una organización de la que, en principio, no son miembros?

La literatura académica ha señalado algunos motivos importantes por los que la gente se implica en proyectos de crowdsourcing. En primer lugar, sin duda, los participantes pueden tener motivaciones extrínsecas ${ }^{36}$, esperando recompensas financieras que incluyen oportunidades de ingresos adicionales, premios en efectivo, parte del valor de una solución ganadora u otras recompensas, como ofertas de trabajo y suministros gratuitos del producto (como fue el caso de Opal, la máquina de nuggets y helados). Segundo, los miembros de la multitud también pueden responder a motivos intrínsecos, como el desarrollo de unas habilidades o unos conocimientos particulares ${ }^{37}$, el reconocimiento personal ${ }^{38}$, el orgullo por una imagen social mejorada y la pura alegría de participar, contribuir en algo concreto y útil ${ }^{39}$. Los motivos también pueden ser mixtos, como los exhibidos por el maestro en Skillshare que ofrecen sus lecciones gratuitas como una forma de construir una marca personal y un canal de promoción para otros productos o servicios ${ }^{40}$.

33 Donati, P. (2001) y (2017).

34 Blustein, D. L. (2011).

35 Goldman, B. L. (2014).

36 Doan, A.; Ramakrishnan, R. y Halevy, A. Y.(2011); Füller, J. (2010); de Beer, J.; McCarthy, I. P.; Soliman, A. y Treen, E. (2017).

37 Täuscher, K. (2017).

38 Jeppersen, L. B. y Frederiksen, L. (2006).

39 Von Ahl,L. y Dabbish, L. (2004).

40 Brabham, D. C. (2010). 
Basadas en estas teorías de la motivación, las empresas plantean diferentes tipos de incentivos en las crowd-calls. Algunas ponen a disposición una compensación económica para los participantes. Es este el caso de Amazon MechanicalTurk, un market place online que "ayuda a las empresas a encontrar personas para realizar tareas. En general, las computadoras son pésimas para identificar elementos en una fotografía, robar documentos reales para encontrar información de identificación, escribir descripciones breves de productos, transcribir podcasts. Amazon llama a las tareas HIT (tareas de inteligencia humana); están diseñados para requerir muy poco tiempo y, en consecuencia, ofrecen muy poca compensación, desde unos centavos hasta unos pocos dólares”" ${ }^{\prime 1}$ Otras calls requieren competencias mucho más especializadas, por lo que ofrecen recompensas más elevadas. EliLilly, DuPont, Boeing y otras empresas usuarias de la plataforma InnoCentive ofrecen pagas de entre $\$ 10.000$ y $\$ 100.000$ por cada solución ${ }^{42}$.

Cuando la participación en crowdsourcing se define como mero intercambio económico las transacciones efectivas resultan habitualmente insuficientes y, con frecuencia, problemáticas. En estos casos el crowdsourcing se convierte fácilmente en "el nuevo depósito de mano de obra barata" ${ }^{43}$, donde participan únicamente aquellos que están en necesidad de trabajar o no encuentran una alternativa profesional mejor. Durante las últimas décadas las empresas han estado buscando en el extranjero, principalmente en India o en China, mano de obra barata. Ahora no importa dónde estén los trabajadores mientras estén conectados a la red: por tanto, los participantes en la call de GE Apliances solo Ismael Ramos y dos finalistas recibieron dinero a cambio de su trabajo. Los profesores que contribuyen con sus cursos al crecimiento de Skillshare pueden no recibir retribución alguna si sus clases no son suficientemente demandadas. Los que ayudaron a Heineken a mejorar el diseño de sus botellas y en el reciclaje de sus envases no percibieron dinero por ello.

En ocasiones, la crowd no es consciente de que hay una empresa que se beneficia de su trabajo. Esto es lo que sucede cuando "Google utiliza los pequeños (y más bien molestos) cuadros de diálogo, que piden a los usuarios que escriban los fragmentos de texto que ven de imágenes distorsionadas en la pantalla. Se cree que esta utilidad web solo sirve para autenticar usuarios humanos. Sin embargo, cada vez que se completa la tarea de teclear caracteres, los indi-

41 Howe, J. (2006), p. 5.

42 Howe, J. (2006), p.3.

43 Howe, J. (2006), p. 2. 
viduos están realmente digitalizando lo que el software de reconocimiento óptico de caracteres (OCR) no ha podido leer. De esta manera, el crowdsourcing de micro tareas ayuda a digitalizar los archivos de The New York Times y la incorporación de manuscritos antiguos a Google Books" ${ }^{44}$.

Si el crowdsourcing se entiende como intercambio económico, es fácil que se convierta en un instrumento de dominación para los agentes con más poder, es decir, aquellas plataformas con vocación monopolística. En esos casos, es fácil que fracase o que provoque rechazo de quienes sienten que están recibiendo una compensación injusta o insuficiente. Si una multitud sospecha que la organización que les convoca obtiene ganancias oportunistas sin reconocer y compensar su propiedad intelectual o emocional, esto puede ser una poderosa razón para que la multitud retenga información o evite la participación por completo. La furiosa reacción que siguió a la mala gestión que Moleskine hizo de las expectativas de la comunidad creativa en las redes sociales pone de relieve que importante es saber gestionar las expectativas de reciprocidad de las personas que conforman la multitud de una organización ${ }^{45}$.

\section{CONSTRUIR RELACIONES}

Las motivaciones y las expectativas de reciprocidad de una crowd no son solo, ni siempre, de índole específica. Las empresas de crowdsourcing más exitosas son aquellas que logran establecer relaciones a largo plazo con y entre los participantes ${ }^{46}$. En este sentido, las tecnologías wiki empresariales (Wikipiedia o Linux Project) que precisan de una cooperación continua para crear y negociar contenidos enriquecidos a partir de conocimientos dispersos, se basan en diferentes tipos de intercambio, relaciones que van más allá de la mera transacción.

En particular, Donati (2001 y 2017) propone que el trabajo, en tanto que relación social, consiste en un vínculo significativo que emerge (se genera y se actualiza) mediante la acción recíproca de quienes participan en una red de intercambios orientados a la producción, distribución y consumo de bienes y servicios de los que depende la vida (el modus vivendi) de los sujetos miembros de esa red.

44 Prpi , J.; Shukla, P. P.; Kietzmann, J. H. y McCarthy, I. P. (2015), p. 79.

45 de Beer, J.; McCarthy, I. P.; Soliman, A. y Treen, E. (2017), pp. 214-215. 
Según el paradigma societario y productivo de la época pre-moderna, el trabajo se entendía como una actividad servil de intercambio con la naturaleza; en la época industrial, era una prestación cualificada para la producción de bienes y servicios, entendidos como "objetos"; en la época digital el trabajo emerge como actividad dirigida a valorizar las relaciones sociales de quien trabaja, en cuanto la actividad se distingue por las cualidades relacionales que puede ofrecer a todos los stakeholders ${ }^{47}$.

En el Crowdsourcing las relaciones que se generan entre participantes se valoran de un modo distinto (y, con frecuencia, mayor) a la transacción de tiempo por dinero. Para ilustrar, MindSumo es una plataforma en línea para resolver problemas creada a partir de Stanford StudentStartupAccelerator, la incubadora de empresas de la Universidad de Stanford. MindSumo se posiciona como una red abierta para el estudiante, un tipo de centro de innovación en línea que ofrece prácticas. Las empresas presentan problemas a los que el alumnado se está enfrentando en un momento dado y los mismos estudiantes formulan soluciones. Aquellas soluciones que se llegan a implantar reciben recompensas en efectivo. Más allá del incentivo monetario, MindSumo enfatiza los eventos temporales que son importantes para los estudiantes. Por ejemplo, vincula los problemas con los cursos relacionados en los cuales están matriculados y con sus futuras carreras. El objetivo principal de MindSumo es orientarles sobre el desarrollo de habilidades profesionales y el trabajo en red como parte de su experiencia académica. Los gerentes de contratación constantemente clasifican la resolución de problemas, las comunicaciones y las habilidades de pensamiento creativo como las competencias más requeridas al contratar nuevos graduados universitarios.

En la sociedad digital, el trabajo se manifiesta cada vez más abiertamente como un nexo relacional, eso es, un vínculo significativo que une a productores, distribuidores y consumidores de bienes y servicios ${ }^{48}$. En tanto que vínculo significativo el trabajo, como toda relación social, incluye una semántica estructural y una semántica referencial ${ }^{49}$. De acuerdo con su misma semántica estructural, el trabajo como relación social supone la conexión, la limitación, el condicionamiento recíproco y la estructura, que a la vez restringe y permite

46 Brabham, D. C. (2010); Fedorenko, I.; Berthon, P. y Rabinovich, T.(2017); Prpi , J.; Shukla, P.; Kietzmann, J. H. y McCarthy, I. P. (2015).

47 Donati, P. (2017), pp. 30-32.

48 Blustein, D. L. (2011).

49 Donati, P. (2011). 
la acción de los trabajadores. En el ejemplo anterior, MindSumo es una plataforma que facilita la conexión con otros miembros de una red de estudiantes y empresas, así como el acceso a problemas concretos y a sus respectivas soluciones. Esta plataforma pretende desarrollar habilidades específicas gracias al trabajo en las cuestiones que se proponen y gracias también a las calificaciones que se otorgan a los estudiantes según las soluciones que ellos mismos ofrecen. A partir de una encuesta interna reciente de 400 estudiantes miembros de MindSumo, el 75\% de ellos ejercitan y perfeccionan las habilidades existentes gracias a los retos, y casi el $45 \%$ informan haber adquirido una nueva habilidad que esperan les ayude en su carrera ${ }^{50}$. Mientras ayuda a los estudiantes a desarrollar sus habilidades, MindSumo también hace posible que las empresas obtengan soluciones a corto plazo y entren en contacto con un grupo de talentos para el futuro. Todos los miembros de la red deben atenerse siempre a las mismas normas de comportamiento y de uso de los recursos disponibles en la plataforma.

De acuerdo con su semántica referencial, el trabajo como relación social atañe a un marco interpretado por los significados simbólicos de diferentes tipos y grados de intencionalidad que están más o menos de acuerdo con los actores involucrados ${ }^{51}$. La principal intención declarada de MindSumo es dirigir a los estudiantes hacia el desarrollo de unas habilidades profesionales concretas. MindSumo muestra a los estudiantes cómo aprovechar su experiencia en el CV y en las entrevistas de trabajo. Por lo tanto, les permite no solo competir por algunas recompensas económicas, sino también construir narrativas biográficas reflexivas para mejorar su identidad ${ }^{52}$, en torno a sus propios proyectos profesionales y personales.

En la sociedad digital el trabajo asalariado propio de la sociedad industrial va siendo reemplazado por un trabajo más autónomo, con sus aspectos positivos y sus aspectos negativos, sus oportunidades y sus riesgos. Ahora crecen las expectativas de un trabajo más creativo y menos dependiente, más flexible y menos rígido, más expresivo y menos instrumental, más valorado por su calidad y menos por su cantidad ${ }^{53}$.

50 MindSumo (2018).

51 Donati, P. (2011), p. 87.

52 Fedorenko, I.; Berthon, P. y Rabinovich, T. (2017), p. 160

53 Donati, P. (2017), p. 69. 
De acuerdo con Donati ${ }^{54}$, en la sociedad actual asistimos a la redefinición del trabajo como una actividad comunicativa significante en redes altamente diferenciadas de producción-distribución-consumo cuya dinámica debemos comprender. Toda dinámica relacional implica el entrelazamiento de su dimensión estructural y su dimensión referencial, es decir, la interacción entre los recursos y las normas propios de la relación, con los objetivos y los valores que le dan sentido.

Numerosas plataformas de crowdsourcing ejemplifican esta dinámica relacional. En ellas, el trabajo emerge como vínculo significativo entre los miembros de una red que intercambian recursos según normas específicas para alcanzar objetivos que consideran importantes para su vida ${ }^{55}$. El sentido de esta dinámica está abierto a la definición compartida de los valores que legitiman los objetivos situacionales, el uso de recursos y las normas de aplicación, sin limitarse a una transacción económica. De la mutua influencia entre recursos, normas, objetivos y valores emerge el tipo de relación propio de cada red. Así pues, toda relación social articula un conjunto de recursos disponibles, normas reguladoras, objetivos prefijados y valores legitimadores propios de esa relación. Las actividades de crowdsourcing generan vínculos significativos entre los miembros de la red en la medida en que combinan recursos y objetivos con normas y valores compartidos por los participantes en una call. De este modo, el trabajo en una red activada por una call, es un trabajo relacional. Las razones del éxito o del fracaso de una call pueden residir en uno o varios de estos cuatro elementos.

a) Recursos-Los recursos empleados en una call pertenecen a la empresa y la misma red. Se trata, sobre todo, de recursos cuyo uso está conectado con los objetivos compartidos de la call (compartidos entre organización y crowd), las normas aceptadas por la red y los valores de la comunidad que surge de la participación. Los modelos comerciales basados en el crowdsourcing pueden crear relaciones a largo plazo con las multitudes si permiten que los contribuyentes crezcan como trabajadores al usar la plataforma. Muchas de las empresas, por lo tanto, proporcionan a los contribuyentes no solo recursos sino también retroalimentación basada en datos manifiestos sobre su desempeño. Los concursos de ideas e innovación han demostrado ser más efectivos si los contribuyentes disponen de una gran transparencia sobre los criterios para ga-

54 Donati, P. (2017), p. 32.

55 Blustein, D. L. (2011); Rodriguez-Lluesma, C., Garcia-Ruiz, P. y Pinto, J. (2021). 
nar dicho concurso. Del mismo modo, las plataformas de aprendizaje basadas en la multitud pueden crear valor para los contribuyentes si se les permite recibir comentarios de los clientes sobre la calidad de sus contribuciones ${ }^{56}$. En el proceso continuado de contribuir en una call y recibir a cambio beneficios de diverso tipo, los participantes son más conscientes de la relación que tienen con la empresa y con los demás miembros de la multitud. El intercambio continuado y valioso de esfuerzo, tiempo, información, aprendizaje, etc., es más aceptable cuando se entiende como un ejercicio de reciprocidad libre.

b) Objetivos - Ser miembros de una crowd da la oportunidad de actuar y lograr resultados que se consideran merecedores del compromiso que se asume. Las plataformas de participación ciudadana, como TheSunlightFoundation y Portland BusProject enfatizan el empoderamiento y el control que crean para los participantes asegurando un gobierno abierto y responsable ${ }^{57}$. Sin embargo, de nada sirve plantear metas interesantes si no se dispone de los recursos necesarios. Los diseñadores creativos, como Zooppa y 99designs, además de albergar abundantes recursos para los miembros, desde libros informativos y podcasts hasta fuentes de música, organizan cursos de capacitación en línea y seminarios web para ellos. La disponibilidad y la adecuación de los recursos mejoran las contribuciones y fortalecen la vinculación de los participantes.

c) Normas - Las multitudes pueden servir como un recurso importante para las empresas y ofrecer recompensas si sus contribuciones son valiosas. Sin embargo, las multitudes no se rigen por leyes que estipulen la propiedad de los derechos del empleador sobre el trabajo creativo desempeñado ${ }^{58}$. Las multitudes desarrollan normas específicas en las que intervienen diversos factores psicológicos y sociales. A menudo, la principal motivación de los participantes es experimentar la campaña o ponerse a prueba con el reto del crowdsourcing, sin la preocupación de recibir ninguna compensación monetaria por su contribución ${ }^{59}$. Cada comunidad a menudo desarrolla también sus propios criterios para sanciones sociales (positivas o negativas). Muchas de las principales plataformas usan herramientas como la gamificación para crear diferencias de

56 Täuscher, K. (2017), p. 241.

57 Fedorenko, I.; Berthon, P. y Rabinovich, T.(2017), p. 160.

58 de Beer, J.; McCarthy, I. P.; Soliman, A. y Treen, E. (2017), p. 208.

59 de Beer, J.; McCarthy, I. P.; Soliman, A. y Treen, E. (2017), p. 215. 
status entre los participantes. Por ejemplo, empresas como SAP, Mofilm, CrowdTapyEyeka recompensan a los clientes por sus contribuciones con "puntuaciones de reputación" para indicar el status que ocupan en relación con otros clientes. En algunas plataformas ciertos contenidos exclusivos están disponibles solo para los clientes que más contribuyen; otros llegan incluso a nombrar a los miembros de la comunidad más destacados para que actúen como administradores de la comunidad ${ }^{60}$.

d) Valores- La participación continuada en las actividades de una crowd propicia entre los participantes la aparición de unas identidades distintivas como miembros de una comunidad. La participación a largo plazo con una plataforma de crowdsourcing particular no solo mejora las competencias de los participantes para resolver problemas de la empresa, sino que también se convierte en una parte integral y cargada de valores de sus identidades ${ }^{61}$. Cuando se desarrollan dichas identidades, la duración y la fuerza de la relación con la empresa y la comunidad aumentan. Asimismo, el sentido de la conexión con una organización influye positivamente en la disposición a participar en las actividades que más le interesen ${ }^{62}$.

Varias razones pueden contribuir a la participación en una convocatoria, como el deseo de conseguir algo de dinero, aprender cosas nuevas o ganar una reputación, pero también, y lo más importante, la participación en una multitud se relaciona con la construcción y la expresión de la propia identidad ${ }^{63}$. Una call puede facilitar el desarrollo de una identidad relacional mediante algunas actividades que faciliten la acción recíproca entre los miembros de la crowd y la organización que promueva la call para hacerles protagonistas del trabajo que desempeñan en red. Por ejemplo, permitir la personalización de objetos, prever oportunidades para el aprendizaje o el desarrollo de competencias, celebrar sus contribuciones, pedir que compartan sus historias o que valoren las aportaciones de otros participantes. De este modo, surgen distinciones y estructuras relacionales como las que señalan a los "miembros más activos" o "la mejor aportación del mes" en foros como hall-of-fame (Mofilm) o showrooms (CreateMyTatoo).

60 Fedorenko, I.; Berthon, P. y Rabinovich, T. (2017), p. 163.

61 Fedorenko, I.; Berthon, P. y Rabinovich, T. (2017), p. 161.

62 de Beer, J.; McCarthy, I. P.; Soliman, A. y Treen, E. (2017), p. 215.

63 Fedorenko, I.; Berthon, P. y Rabinovich, T. (2017), p. 156. 
Las empresas de crowdsourcing brindan oportunidades poderosas para que los participantes desarrollen relaciones a largo plazo y generen identidades como miembros de unas comunidades específicas. La participación en la call depende del sentido que esta implicación tenga para el miembro potencial: cuanto mayor es el significado, más probabilidades hay de que un sujeto participe. Para comprender la dinámica de este mercado laboral, es vital comprender hasta qué punto importan los incentivos no pecuniarios ${ }^{64}$, implicarse en una relación significativa con una comunidad donde se crean bienes (materiales e/o inmateriales) que los miembros consideran importantes para sus vidas.

\section{EL TRABAJO RELACIONAL DE LA MULTITUD COMO EMERGENTE EFECTO DE RED}

La transformación actual del trabajo se debe entender como el resultado emergente de un conjunto de variables estructurales y culturales, conectadas entre sí. Los cambios que se producen se originan en cada dimensión del trabajo. Por ejemplo, los recursos disponibles aumentan gracias a los avances tecnológicos. Los objetivos y los contenidos del trabajo se modifican debido a nuevos negocios digitales, o a la re-definición de las carreras profesionales en términos de una mayor movilidad geográfica y sectorial. La regulación normativa del trabajo está pendiente de las nuevas exigencias del derecho global y de la "uberización" del trabajo. Los criterios que las personas emplean para juzgar su trabajo varían según las crecientes demandas de conciliación entre la vida laboral, familiar y personal. Estas dimensiones están conectadas entre sí, pero no en un sentido único, predeterminado.

El éxito (o el fracaso) de una crowdsourcing call no depende solo, ni primariamente, de los recursos tecnológicos disponibles y tampoco de la distribución o del acceso a tales recursos. El crowdsourcing no se puede explicar únicamente desde un punto de vista estructural, como intercambio de intereses. Como hemos visto, es preciso tener en cuenta también la dimensión cultural, el significado identitario, que conlleva para los miembros de la crowd. Implicarse en una call significa comprometerse con una realidad sui generis: una relación entre una organización y una multitud cuyos objetivos y significados son centrales en la existencia de quienes decidan implicarse. En otras palabras,

64 Chandler, D. y Kapelner, A. (2013), p. 132. 
una call tiene un valor relacional cuando no es intercambiable por cualquier otra call.

Muchos de los que aportan ideas para la decoración de Starbucks no las aportarían para otra cadena, pues su participación se explica porque comparten la misión y los valores de esa cadena. Muchos de los que sugieren diseños para camisetas no los aportarían para otros productos, pues su vínculo depende de su afinidad con el mundo de la moda. Muchos de los que participan en MindSumo porque surgió en Stanford no lo harían en otras plataformas menos exclusivas, etc.

Los participantes tienen entonces conciencia de que la crowd es una realidad relacional que emerge como consecuencia de una respuesta suficientemente amplia de personas con recursos, objetivos y motivos propios y acordes con una organización. La literatura ha señalado la importancia del "efecto de red" en la economía colaborativa ${ }^{65}$. Los ejemplos son numerosos. Waze es una aplicación más valiosa que otros mapas online de tráfico porque cada usuario de esta red continuamente aporta datos que ayudan a los demás participantes a conocer el estado del tráfico en tiempo real y tomar decisiones acertadas acerca de su movilidad. Sin una contribución permanente esa información tan útil no existiría. Con todo, las redes generan bienes no solo por agregación sino también como resultado emergente de las relaciones entre sus miembros.

La crowd es capaz de generar bienes relacionales no accesibles a sus miembros como individuos ni tampoco a la organización. Gracias al intercambio y a la capacidad de compartir conocimiento, la crowd movilizada por InnoCentive puede resolver más problemas, y más complicados, que individuos u organizaciones altamente especializadas. Los miembros de la crowd se benefician de esos bienes cuando se implican en la relación. "En el crowdsourcing, las habilidades del consumidor, el conocimiento y otros recursos se aprovechan como una comunidad (es decir, multitud) para crear valor, no solo para la empresa focal sino también para el cliente. De hecho, es un valor determinado por el cliente lo que impulsa el compromiso y la contribución, y en última instancia influye en el éxito de una empresa de crowdsourcing" ${ }^{66}$. Asimismo, esos bienes solo existen si los individuos participan, si deciden implicarse en la relación dentro de un marco de reciprocidad y transparencia.

65 Entre otros: Castells, M. (2005); Hodgson, G. M. (2016); Hidalgo, A. (2018).

66 Fedorenko, I.; Berthon, P. y Rabinovich, T. (2017), p. 156. 
Tales bienes relacionales resultan de un gobierno local abierto y dialogante, promovido por redes como TheSunlightFoundationo Portland BusProject; o como el acceso a un foro para compartir experiencias de diseño creativo en Threadless, Zooppa, y 99design.La emergencia de estos bienes relacionales depende de la transformación de la crowd en community (comunidad), que a su vez depende de la reflexividad relacional.

Esta reflexividad consiste en la orientación de los sujetos no hacia los objetivos, los recursos o los otros miembros de la relación, sino hacia la relación misma, en la medida en que se considera algo valioso que merece la pena cuidar, o al menos una realidad que les influye y atrae ${ }^{67}$. Hay diversas formas de reflexividad relacional. Una de ellas, la meta-reflexividad es la manera en los miembros de una red que "redefinen continuamente las propias metas y valores y buscan nuevos medios y nuevas reglas para perseguir las actividades con que uno se puede realizar en relación con los otros, para producir bienes comunes que corresponden a valores inmateriales" ${ }^{68}$. Este es probablemente uno de los factores que explican la aparición de videos negativos sobre el Chevrolet Tahoe, criticando su excesivo consumo y exigiendo coches más sostenibles.

Queda pendiente saber en qué medida se puede considerar una crowd como una subjetividad social, en tanto que es un "conjunto de personas que participan en una relación que perciben y ejercen como referencia simbólicointencional y como vínculo estructural, con un valor y una realidad propia" ${ }^{9}$. Ciertamente, las organizaciones se dirigen a una crowd como a un sujeto (colectivo), les encargan unas tareas (outsourcing) que antes daban a individuos o a otras organizaciones: una campaña de publicidad para el Tahoe, el diseño de la nueva botella de cerveza Heineken, una investigación de mercado para la máquina de hielo Opal, etc. Quien realiza ese trabajo es una crowd, donde la interacción continuada es parte esencial. GE no produjo Opal solo gracias a la inteligencia de Ismael Ramos, sino también y sobre todo, gracias a la participación de una red de individuos y grupos dispuestos a implicarse en el desarrollo del prototipo Opal. Además, hemos visto que la crowd es capaz de "conferir identidades sociales a sus miembros" y de actuar "como un sujeto a la búsqueda de un bien común relacional" ${ }^{\prime 70}$. En cambio, no parece que las crowds

67 Donati, P. (2011), p. xvi.

68 Donati, P. (2017), p. 54.

69 Donati, P. (1993), p. 219.

70 Donati, P. (1993), p. 204. 
se comporten como "un actor solidario autónomo, centro de libertad y responsabilidad" $"$.

A partir de aquí, se plantean otras cuestiones para la investigación futura sobre este fenómeno. En primer lugar, está la cuestión de los derechos de propiedad intelectual ${ }^{72}$. Si se considera la crowd como el sujeto que realiza el trabajo, ¿de quién son los derechos de propiedad de las ideas, los diseños, los prototipos, etc., que se logran? En segundo lugar, en la economía de redes se desdibujan las fronteras entre quienes son empleados y quienes no de una organización; y también entre quienes son productores, distribuidores y consumidores. Desde la sociología del consumo se ha propuesto la figura del prosu$m e r^{73}$ como un nuevo paradigma para la sociedad de redes. En la sociología del trabajo, la perspectiva relacional puede aportar una nueva manera de entender la relación entre trabajadores y consumidores cuando ambos roles coinciden en las redes de la economía colaborativa ${ }^{74}$. Por último, sería interesante estudiar otras formas emergentes de trabajo que pueden ser consideradas como "trabajo relacional", en particular algunas formas de co-working y algunas nuevas organizaciones ${ }^{75}$ que incluyen mercados internos de ideas, proyectos y recursos ${ }^{76}$.

\section{CONCLUSIONES}

De acuerdo con la teoría relacional, el trabajo es un vínculo significativo que emerge (se genera y se actualiza) mediante la acción recíproca de quienes participan en una red de intercambios orientados a la producción, distribución y consumo de bienes y servicios de los que depende la vida de los sujetos que integran esa red. Trabajar genera interdependencia e intercambios específicos, de acuerdo con expectativas compartidas, que responden a criterios de valor propios de la relación que une a quienes trabajan con los que se benefician de ese trabajo. El trabajo, en tanto que relación social, tiene varias dimensiones conectadas entre sí: (a) uso de los recursos disponibles, (b) para alcanzar objetivos determinados, (c) según normas reguladoras de la actividad, (d) legitimadas por ciertos criterios de valor.

71 Donati, P. (1993), p. 204.

72 de Beer, J.; McCarthy, I. P.; Soliman, A. y Treen, E. (2017).

73 Ritzer, G. y Jurgenson, N. (2010).

74 Blustein, D. L. (2011).

75 Spinuzzi, C. (2012); Rodriguez-Lluesma, C.; Garcia-Ruiz, P. y Pinto, J. (2021).

76 Lee, M.Y. y Edmondson, M. C. (2017); Malone, T. H. (2004). 
En las últimas décadas, en las sociedades occidentales más avanzadas, se ha estado dando un proceso de "diferenciación relacional" del trabajo: la relación que vincula a trabajadores y beneficiarios del trabajo ya no se entiende única y llanamente como "tiempo a cambio de salario". Se están afirmando otras formas de trabajar, en un marco de reciprocidad e interdependencia entre quienes trabajan y quienes reciben el bien o servicio resultante de ese trabajo, que responden a otros criterios de valor.

El crowdsourcing es un ejemplo significativo de trabajo relacional. Se trata de una llamada online para que un grupo de personas complete una tarea de forma voluntaria, poniendo a disposición sus conocimientos (expertise) y sus propios recursos. Las empresas emplean el crowdsourcing para actividades tan diferentes como la generación de ideas; la resolución de problemas; la discusión, la evaluación y la selección de posibles alternativas; la predicción de los escenarios futuros; el diseño, la fabricación y la financiación de productos innovadores. En respuesta a esa llamada, personas anónimas (profesionales o no) aportan su trabajo, tiempo, creatividad y competencias, sin recibir una compensación a cambio que sea cierta o pactada previamente.

Su emergencia como forma de trabajo depende, por una parte, de factores estructurales como la disponibilidad de unos recursos tecnológicos apropiados; su éxito depende también de factores culturales, como el significado que comporta para los miembros de la crowd. Implicarse en una convocatoria significa comprometerse con una realidad particular: una relación entre una organización y una multitud de personas cuyo objetivo y valor es importante para la vida de quien se implica. Además del intercambio instrumental a partir de intereses diversos, en el despliegue concreto del crowdsourcing asumen relevancia también la construcción de identidades, la reflexividad y la generación de bienes relacionales.

Entre la variedad de significados que los participantes dan a su participación en el crowdsourcing, se cuenta el deseo de aprender cosas nuevas y ganar una reputación, así como la posibilidad de expresar una identidad personal y social ligada a su propia vocación o reputación. Como se ha dado cuenta en el análisis aquí realizado, estos participantes actúan como miembros de una comunidad dentro de la cual se desarrollan relaciones específicas. Emerge, pues, que gracias al crowdsourcing las habilidades y los conocimientos de los participantes se comparten para crear valor, pero no solamente para la empresa sino también para ellos mismos. De hecho, este intercambio impulsa el compromiso y la contribución desde una perspectiva de mutualidad, espontaneidad y corresponsabilidad: todos animados por una dedicación voluntaria. Por todo 
ello, podemos concluir que el crowdsourcing es una de las mejores expresiones de las nuevas formas de trabajo propias de la sociedad post-moderna y se puede comprender mejor si se observan desde una perspectiva relacional.

\section{BIBLIOGRAFÍA}

Andreoni, James (2007), "Giving Gifts to Groups: How Altruism Depends on the Number of Recipients", Fournal of Public Economics, vol. 91, n ${ }^{\circ} 9$, pp. 1731-1749.

Brabham, Daren C. (2010), "Moving the Crowd at Threadless: Motivations for Participations in a Crowdsourcing Application", Information, Communication \& Society, vol. 13, $\mathrm{n}^{\mathrm{o}}$ 8, pp. 1122-1145.

Blustein, David L. (2011), "A Relational Theory of Working”, fournal of Vocational Behavior, vol. 79, $\mathrm{n}^{\circ}$ 1, pp. 1-17.

Brunswicker, Sabine; Bilgram, Volker y Fueller, Johan (2017), “Taming Wicked Civic Challenges with an Innovative Crowd", Business Horizons, vol. $60, \mathrm{n}^{\circ} 2$, pp. 167-177.

Brynjolfsson, Erick y McAfee, Andrew (2014), The Second Machine Age: Work, Progress, and Prosperity in a Time of Brilliant Technologies, WW Norton \& Company, Nueva York.

Castells, Manuel (2005), La era de la información. La sociedad red (nueva edición), Alianza Editorial, Madrid.

Chandler, Dana y Kapelner, Adam (2013), "Breaking Monotony with Meaning: Motivation in Crowdsourcing Markets", Journal of Economic Bebavior \& Organization, vol. 90, pp. 123-133.

de Beer, Jeremy; McCarthy, Ian P.; Soliman, Adam y Treen, Emily (2017), "Click Here to Agree: Managing Intellectual Property when Crowdsourcing Solutions”, Business Horizons, vol. 60, n² 2, pp. 207-217.

De Masi, Domenico (2002), Ozio creativo, Rizzoli, Milán.

Doan, Anhai; Ramakrishnan, Raghu y Halevy, Alon Y. (2011), "Crowdsourcing Systems on the World-wide Web", Communications of the ACM, vol. $54, n^{\circ} 4$, pp. 86-96. 
Donati, Pierpaolo (1993), La cittadinanza societaria, Laterza, Bari-Roma.

Donati, Pierpaolo (2001), Il lavoro che emerge: prospettive del lavoro come relazione sociale in una economia dopo-moderna, Bollati Boringhieri, Milán.

Donati, Pierpaolo (2006), Repensar la sociedad, Eiunsa, Madrid.

Donati, Pierpaolo (2010), Relational Sociology: a New Paradigm for the Social Sciences, Routledge, Londres.

Donati, Pierpaolo (2017), Quale lavoro? L'emergere di una economía relazionale, Marietti, Génova.

Donati, Pierpaolo y Archer, Margaret S. (2015), The Relational Subject, Cambridge University Press, Cambridge, MA.

Donati, Pierpaolo y García Ruiz, Pablo (2021) Sociología Relacional. Una lectura de la sociedad emergente, Prensas Universitarias de Zaragoza.

Emirbayer, Mustafa (1997), "Manifesto for a Relational Sociology", American Fournal of Sociology, vol. 103, $\mathrm{n}^{\mathrm{o}}$ 2, pp. 281-317.

Estellés-Arolas, Enrique y González-Ladrón de Guevara, Fernando (2012), "Towards an Integrated Crowdsourcing Definition", Fournal of Information Science, vol. 38, n² 2, pp. 189-200.

Fedorenko, Ivan; Berthon, Pierre y Rabinovich, Tamara (2017), "Crowded Identity: Managing Crowdsourcing Initiatives to Maximize Value for Participants Through Identity Creation", Business Horizons, vol. 60, n² 2, pp. 155165.

Füller, Johann (2010), "Refining Virtual co-Creation from a Consumer Perspective", California Management Review, vol. 52, n 2, pp. 98-122.

Goldman, Brian Leonel (2014). "El crowdsourcing como forma de apropiación de valor en el capitalismo informacional", Revista Hipertextos, vol. 1, no 2, pp. 131-165.

Hidalgo Manuel, Alejandro (2018), El empleo del futuro. Un análisis del impacto de las nuevas tecnologías en el mercado laboral, Ediciones Deusto, Bilbao.

Hines, Andy (2011), "A Dozen Surprises about the Future of Work", Employment Relations Today, vol. 38, n 1, pp. 1-15.

Hodgson, Geoffrey M. (2016), "The Future of Work in the Twenty-First Century", Fournal of Economic Issues, vol. 50, n 1, pp. 197-216. 
Howe, Jeff (2006), “The Rise of Crowdsourcing”, Wired Magazine, vol. $14, n^{\circ} 6$, pp. 1-5.

ICEMD (2018), Colaboración y co-creación de usuarios y consumidores. http://blogs.icemd.com/blog-colaboracion-y-co-creacion-de-usuarios-yconsumidores-crowdsourcing/caso-de-exito-en-crowdsourcing-my-starbucksidea/ (Accessed 19/01/2018).

Jeppesen, Lars Boy Frederiksen, Lars (2006), "Why do Users Contribute to Firm-Hosted User Communities? The Case of Computer-Controlled Music Instruments", Organization Science, vol. 17, n 1, pp. 45-63.

Khallash, Sally y Kruse, Martin (2012), "The Future of Work and WorkLife Balance 2025”, Futures, vol. 44, n 7, pp. 678-686.

Kietzmann, Jan H. (2017), "Crowdsourcing: A Revised Definition and Introduction to New Research”, Business Horizons, vol. 60, n 2, pp. 151-254.

Lee, Michael Y. y Edmondson, Amy C. (2017), "Self-managing Organizations: Exploring the Limits of less-hierarchical Organizing", Research in Organizational Behavior, vol. 37, pp. 35-58.

Lips-Wiersma, Marjolein y Morris, Lani (2017), The Map of Meaning: A Guide to Sustaining our Humanity in the World of Work, Routledge, Londres.

Malone, Thomas W. (2004), The Future of Work: How the New Order of Business will shape your Organization, your Management Style and your Life, HBS Press, Cambridge, MA.

McAfee, Andrew y Brynjolfsson, Erik (2017), Machine, Platform, Crowd: Harnessing our Digital Future, WW Norton \& Company, Nueva York.

McKinsey \& Co. (2017), "Jobs: WorkforceTransitions in a Time of Automation", enhttps://www.mckinsey.com/global-themes/future-of-organizations-and-work/what-the-future-of-work-will-mean-for-jobs-skills-and-wages (Accesed 15/01/2019).

MindSumo (2018), Official website. https://www.mindsumo.com/challenges (Accessed 17/01/2019).

Prpi , John; Shukla, Prasant P.; Kietzmann, Jan H. y McCarthy, Ian Paul (2015), "How to Work a Crowd: Developing through Crowdsourcing", Business Horizons, vol. 58, $\mathrm{n}^{\circ} 1$, pp. 77-85. 
Ritzer, George y Jurgenson, Nathan (2010), "Production, Consumption, Prosumption: The Nature of Capitalism in the Age of the Digital Prosumer", Fournal of Consumer Culture, vol. 10, nº 1, pp. 13-36.

Rosso, Brent D.; Dekas, Kathryn H. y Wrzesniewski, Amy (2010), "On the Meaning of Work: A Theoretical Integration and Review", Research in Organizational Behavior, vol. 30, pp. 91-127.

Schwartz, David (2018), "Embedded in the Crowd: Creative Freelancers, Crowdsourced Work, and Occupational Community", Work and Occupations, vol. $45, \mathrm{n}^{\circ} 3$.

Spinuzzi, Clay (2012), "Working Alone Together: Coworking as Emergent Collaborative Activity". Fournal of Business and Technical Communication, vol. 26, n 4 , pp. 399-441.

Rodriguez-Lluesma, Carlos; García Ruiz, Pablo y Pinto, Javier (2021), "The Digital Transformation of Work. A Relational View", Business Ethics. A European Review, vol. 30, n 1, pp. 157-167.

Täuscher, Karl (2017), "Leveraging Collective Intelligence: How to Design and Manage Crowd-Based Business Models". Business Horizons, vol. 60, ${ }^{\circ}$ 2, pp. 237-245.

Von Ahn, Laura y Dabbish, Luis (2004), "Labeling Images with a Computer Game", en Proceedings of the SIGCHI Conference on Human Factors in Computing Systems, pp. 319-326.

Wilson, Matthew; Robson, Karen y Botha, Elsamari (2017), "Crowdsourcing in a Time of Empowered Stakeholders: Lessons from Crowdsourcing Campaigns", Business Horizons, vol. 60, nº 2, pp. 247-253. 